\title{
VORWORT ZUR 1. AUFLAGE
}

Die außerordentlich zustimmende Aufnahme, die Richard Vogels Werk über das Gebiet Königstein allgemein fand, läßt das dringende Bedürfnis für eine umfassende Darstellung der heimatlichen Werte in kleinen, begrenzten Gebieten erkennen. Dem von vielen Seiten geäußerten Wunsch nach baldiger Fortführung der Reihe kommt nunmehr Hermann Lemmes Bearbeitung der hinteren Sächsischen Schweiz entgegen. Die Beschreibung dieser eigenwilligen Landschaft mit ihrem Gewirr von Felsen und Schlüchten erfordert einen guten, mit jedem Weg vertrauten Kenner, der auch um die schmalen Felspfade weiß und sie selbst gegangen ist, wie sie sonst nur beherzten Kletterern vertraut sind; sie verlangt einen Heimaterzieher, der Jugend und Erwachsene für die Fülle oft auch herber Schönheiten zu begeistern vermag. Die Aufnahme im linkselbischen Gebiet um Schöna und um die Zschirnsteine führte Dr. Gerhard Engelmann durch.

Eine landschaftliche Zusammenschau unter fachlichen Gesichtspunkten wird für die gesamte Sächsische Schweiz von berufenen Vertretern der wissenschaftlichen Institute gegeben, die das Unternehmen einer heimatkundlichen Bestandsaufnahme verantwortlich mit tragen. Sie erscheint in Verbindung mit der Darstellung des Gebietes um Rosenthal im folgenden 3. Bande der Reihe. Deshalb konnte sich die vorliegende Arbeit auf eine knappe Einführung in das behandelte Gebiet beschränken.

Die redaktionelle Betreuung des Manuskripts übernahm im Auftrage der Kommission für Heimatforschung Herr Dipl.-Geogr. Dietrich Zühlke. Die letzte Fassung kam unter sachkundigem Rat, stofflicher Unterstützung und gutachtlicher Billigung mehrerer Fachvertreter zustande.

Insbesondere sei gedankt Herrn Prof. Dr. Edgar Lehmann, Leipzig, sowie Herrn Dr. Gotthold Weicker, Dresden, und Herrn Dipl.-Geogr. Gottfried Andreas, Pirna, als den Vertretern der Geographie; Herrn Dr. Hans Prescher für seinen unermüdlichen Einsatz bei der Klärung geologischer Fragen; den Herren Max Militzer, Dresden, und Hans Förster, Papstdorf, für die Überprüfung von Angaben zur Biologie und zum Naturschutz; Herrn Dr. Werner Coblenz für Angaben zur Vorgeschichte, Herrn Dr. Alfred Opitz für Fragen zur Geschichte der Arbeiterbewegung, Herrn Dr. Karl-Heinz Blaschke, Dresden, für ortsgeschichtliche und den Herren Dr. H. Walther und Dr. Ernst Eichler, Leipzig, für namenkundliche Hinweise. In dankenswerter Weise stellten Fräulein Elisabeth Hütter und Herr Dr. Heinrich Magirius Beschreibungen der Bau- und Kunstdenkmäler, Herr Architekt Oskar Pusch Angaben zur Rekonstruktion der alten 
Raubburgen, Herr Dr. Alfred Fiedler ergänzendes Material zu den Hausformen, Herr Dr. Johannes Georgi und Frau Margarethe Petraschk Material zur Sebnitzer Volkskunst zur Verfügung. Herr Gerhardt Müller war in bewährter Weise um die übrigen volkskundlichen Beiträge besorgt.

Sie unterstützten alle das Bemühen der Bearbeiter, im Sinne eines neuen Heimatbewußtseins in die Wesenszüge und Sonderheiten einer einzigartigen Landschaft einzuführen und weite Kreise mit ihren intimen Schönheiten vertraut zu machen.

Für die Kommission für Heimatforschung Prof. Dr. Ernst Neef

\section{VORWORT ZUR 2. AUFLAGE}

Nachdem die 1. Auflage des Bandes rasch vergriffen war, erschien die Herausgabe einer 2. Auflage angebracht, um die ständig anhaltende Nachfrage befriedigen zu können. Da eine völlige Neubearbeitung nicht notwendig war und das Buch den zahlreichen Besuchern dieses Gebietes recht bald wieder zur Verfügung stehen sollte, haben sich Herausgeber und Verlag zu einem seitengetreuen Nachdruck entschlossen. Wenn dieser auch größere Änderungen ausschließt, so konnten die aus verschiedenen Kreisen eingegangenen Hinweise und Berichtigungen, für die an dieser Stelle gedankt sei, doch weitgehend berücksichtigt werden.

Ernst Neef 